\title{
Scénarios de modélisation de l'épidémie de COVID-19 au Canada
}

\author{
Nick H Ogden ${ }^{1 \star}$, Aamir Fazil1, Julien Arino², Philippe Berthiaume1, David N Fisman³, Amy L Greer ${ }^{4}$, \\ Antoinette Ludwig', Victoria Ng${ }^{1}$, Ashleigh R Tuite ${ }^{3}$, Patricia Turgeon ${ }^{1}$, Lisa A Waddell', \\ Jianhong $\mathrm{Wu}^{5,6}$
}

\section{Résumé}

Contexte : Le virus du syndrome respiratoire aigu sévère 2 (SARS-CoV-2), un coronavirus ayant probablement comme origine la chauve-souris, s'est transmis des animaux sauvages vers les humains en Chine à la fin de 2019 se manifestant sous la forme d'une maladie respiratoire. La maladie du coronavirus 2019 (COVID-19) s'est propagée d'abord en Chine, puis à l'échelle mondiale, entrainant une pandémie.

Objectifs : Cet article décrit la modélisation prédictive de la COVID-19 en général et les efforts déployés au sein de l'Agence de la santé publique du Canada pour modéliser les effets des interventions non pharmaceutiques (INP) sur la transmission du SARS-CoV-2 dans la population canadienne afin d'appuyer les décisions en santé publique.

Méthodes : Les objectifs généraux de deux approches de modélisation, 1) un modèle à base d'agent et 2) un modèle compartimenté déterministe sont décrites et un synopsis des études est illustré à l'aide d'un modèle élaboré à partir du logiciel Analytica 5.3.

Résultats : Sans intervention, plus de $70 \%$ de la population canadienne pourrait être infectée. Les interventions non pharmaceutiques, appliquées avec une intensité insuffisante pour éteindre l'épidémie, réduisent le taux d'attaque à $50 \%$ ou moins, et l'épidémie est plus longue avec un pic moins élevé. Si les INP sont retirées tôt, l'épidémie pourrait reprendre de la force, ce qui se traduirait par des pourcentages élevés (plus de $70 \%$ ) de la population touchée. Si les INP sont appliquées avec une intensité suffisamment élevée pour éteindre l'épidémie, le taux d'attaque peut être réduit entre $1 \%$ et $25 \%$ de la population.

Conclusion : Appliquer les INP avec une intensité suffisamment élevée pour éteindre l'épidémie semblerait être le choix privilégié. Le retrait des INP perturbatrices, comme les fermetures, doit être accompagné d'améliorations aux autres INP afin de prévenir de nouvelles éclosions, d'identifier et de contrôler toute nouvelle chaîne de transmission.

Citation proposée : Ogden NH, Fazil A, Arino J, Berthiaume P, Fisman DN, Greer AL, Ludwig A, Ng V, Tuite AR, Turgeon P, Waddell LA, Wu J. Scénarios de modélisation de l'épidémie de COVID-19 au Canada. Relevé des maladies transmissibles au Canada 2020;46(6):225-31. https://doi.org/10.14745/ccdr.v46i05a08f

Mots-clés : modélisation, COVID-19, Canada, interventions non pharmaceutiques
Cette oeuvre est mise à la disposition selon les termes de la licence internationale Creative Commons Attribution 4.0

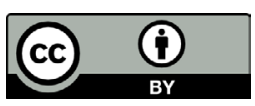

Affiliations

${ }^{1}$ Division des sciences des risques pour la santé publique, Laboratoire national de microbiologie, Agence de la santé publique du Canada, SaintHyacinthe (QC) et

Guelph, ON

2 Département des mathématiques et des sciences des données NEXUS, Université du Manitoba, Winnipeg, MB

${ }^{3}$ Dalla Lana School of Public Health, Université de Toronto, Toronto, ON

${ }^{4}$ Department of Population Medicine, Université de Guelph, Guelph, ON

${ }^{5}$ Laboratory for Industrial and Applied Mathematics, Département de mathématiques et de statistiques, Université York, Toronto, ON

${ }^{6}$ Fields-CQAM Laboratory of Mathematics for Public Health, Université York, Toronto, ON

\section{${ }^{\star}$ Correspondance :}

nicholas.ogden@canada.ca

\section{Introduction}

Dans cet article, nous passons en revue les efforts déployés au sein de l'Agence de santé publique du Canada (ASPC) pour modéliser la transmission du virus du syndrome respiratoire aigu sévère 2 (SARS-CoV-2), l'agent de la maladie à coronavirus 2019 (COVID-19), afin d'étayer les décisions de santé publique. La pandémie de COVID-19 s'est propagée à une vitesse remarquable. Le SARS-CoV-2 est probablement un coronavirus provenant de la chauve-souris (1) qui peut s'être transmis aux humains à partir d'un réservoir animal intermédiaire qui n'est pas encore identifié. Le premier événement d'exposition humaine détecté était lié à un marché traditionnel de produits frais dans la ville de Wuhan, la capitale de la province du Hubei, en Chine, vers la fin de 2019 (2). Le virus était probablement déjà capable de se transmettre entre humains, mais sa transmissibilité a évolué de manière plus efficace vers la fin de 2019 (3). La transmission entre humains a été officiellement reconnue par la communauté mondiale de la santé publique à la mi-janvier 2020 (4). Peu de temps après, une propagation géographique en Chine 
était signalée. Des mesures intensives de santé publique (ou interventions non pharmaceutiques; INP) de détection des cas, de dépistage des contacts, de mise en quarantaine et de distanciation physique (sociale) ont été mises en œuvre dans la province du Hubei, et la région a été isolée du reste de la Chine par des restrictions de voyage (5). Des restrictions sur les voyages internationaux à destination et en provenance de la Chine ont été introduites, mais des cas s'étaient déjà répandus à l'extérieur de la Chine (6), et la propagation mondiale qui en a résulté a entraîné la déclaration d'une pandémie (4). Les premiers cas liés aux voyages ont été recensés au Canada en janvier 2020 et, en avril 2020, la transmission communautaire avait lieu dans toutes les provinces, à l'exception peut-être de l'île-du-PrinceÉdouard. Les territoires n'ont pas encore signalé de transmission communautaire. La majorité des cas et des décès ont été signalés dans les quatre plus grandes provinces (ColombieBritannique, Alberta, Ontario et Québec). La distanciation physique (notamment les fermetures d'écoles, de collèges, d'universités et d'entreprises "non essentielles ») a été mise en œuvre à partir de la mi-mars 2020 au Canada, et les réductions subséquentes de la transmission de la maladie suggèrent que ces mesures et d'autres INP (détaillées ci-dessous) ralentissent l'épidémie (7).

\section{Évolution de la modélisation de la COVID-19}

Dans les débuts, les études de modélisation portaient sur l'épidémie en Chine, particulièrement sur la dynamique de l'épidémie dans la ville de Wuhan et dans toute la province du Hubei. À ce stade précoce, beaucoup d'efforts ont été déployés pour analyser les données de surveillance de la Chine afin d'obtenir des estimations des paramètres comme le taux de reproduction de base $\left(R_{0}\right)$, le taux de mortalité et la période $d$ 'incubation (8). Pour les premières tentatives de modèles dynamiques de type SEIR (Susceptible-Exposé-InfectieuxRétablis), les estimations des paramètres ont été " empruntées" à ce qui était connu au sujet d'autres coronavirus (SARS-CoV et MERS-CoV) (6) et/ou obtenues en adaptant les modèles aux données de surveillance $(9,10)$. À mesure que d'autres données sur la transmission du virus du SARS-CoV-2 et l'évolution de I'infection chez les humains sont devenues disponibles, les paramètres des modèles sont devenus de plus en plus ajustés à l'aide de données propres au SARS-CoV-2 (11). Avec la propagation mondiale de la maladie, et considérant que le vaccin ne sera probablement pas disponible avant encore un an, les efforts de modélisation se sont portés vers l'évaluation de l'étendue possible de la pandémie dans des pays en dehors de la Chine, et l'impact des différentes INP $(11,12)$. Des données scientifiques émergentes ont révélé que le virus SARS-CoV-2 est hautement transmissible par voie respiratoire et possiblement par voie féco-orale, qu'il est transmis avant l'apparition des symptômes et que certains cas peuvent être entièrement asymptomatiques $(13,14)$. Le virus peut être hautement pathogène pour les personnes âgées et certaines personnes plus jeunes, en particulier celles avec des comorbidités (14). La transmission présymptomatique, les symptômes bénins (en particulier chez les groupes d'âge plus jeunes) et les cas asymptomatiques nuisent tous à la détection des cas infectieux (contrairement au SARS) (15), ce qui rend le contrôle difficile. Les efforts de modélisation déployés à ce jour ont mis en lumière l'ampleur du défi auquel nous sommes confrontés : 1) la population mondiale est totalement naïve immunologiquement; 2) le virus est hautement transmissible (les valeurs $R_{0}$ peuvent être supérieures à cinq dans certains contextes) (16) et 3) le niveau de pathogénicité du SARS-CoV-2 signifie que même les systèmes de santé les plus avancés au monde peuvent être complètement dépassés si le virus se propage sans introduire d'INP. Par ailleurs, contrairement à l'influenza pandémique, il n'existe aucun antiviral efficace connu.

\section{Modélisation de la COVID-19 au Canada}

La modélisation prédictive de la COVID-19 par des scientifiques canadiens est un domaine d'étude qui datait d'environ trois mois au moment de la rédaction (début de mai 2020), mais il existe au Canada de grandes compétences en modélisation de la transmission des maladies infectieuses. Certains modèles précédemment développés qui étudiaient les interventions pour contrôler la grippe H1N1 et d'autres grippes $(17,18)$ ont été adaptés pour évaluer la transmission du SARS-CoV-2 et les impacts de différentes INP $(9,19,20)$. Tang et coll. (9) et Li et coll. (21) ont développé des modèles de type SEIR pour explorer la transmission et tirer des leçons des INP mises en œuvre en Chine et en Corée du Sud. Un groupe d'experts en modélisation, composé de plus de 50 modélisateurs et épidémiologistes fédéraux, provinciaux, territoriaux et universitaires, a été mis sur pied par I'ASPC pour élaborer un réseau canadien de modélisation de la COVID-19 qui appuie la prise de décisions. Des groupes semblables ont été mis sur pied dans d'autres pays, et la liaison entre eux a été facilitée par un groupe de modélisation de l'Organisation mondiale de la Santé.

\section{Modélisation de la COVID-19 par I'ASPC}

En janvier 2020, un groupe de modélisation a été formé afin d'entreprendre l'élaboration de deux approches de modélisation complémentaires. Le principal objectif de cette modélisation était d'évaluer l'impact des différentes INP et les niveaux d'efficacité nécessaires pour contrôler l'épidémie au Canada. En l'absence d'un vaccin ou d'un traitement, les INP disponibles pour contrôler l'épidémie, qui ont été explorées dans la modélisation, sont 1) la distanciation physique (fermeture d'écoles, de collèges et d'universités, lieux de réunion, rassemblements et distanciation personnelle) qui réduit les taux de contact entre les membres de la société (notamment ceux qui 
peuvent être infectés), 2) la détection des cas par la surveillance et leur isolement pour les empêcher de transmettre l'infection et 3) le suivi et la mise en quarantaine des personnes qui ont été en contact avec des cas.

Les deux approches de modélisation utilisées seront publiées sous forme d'articles distincts et plus détaillés et sont appelées " approches » étant donné que les modèles eux-mêmes ont évolué avec l'amélioration des connaissances. Ce qui suit est une description générale de ces approches, qui sont fondées sur 1) un modèle fondé sur les agents (22) et 2) un modèle de compartiment déterministe (23). L'approche fondée sur les agents a été élaborée de novo à l'aide du logiciel Anylogic ${ }^{\odot}$, tandis que le modèle déterministe s'exécute dans $R$ (24). Les versions initiales du modèle déterministe ont été adaptées de Tang et coll. (9). Les deux sont des modèles de type SEIR avec des éléments pour modéliser le SARS-CoV-2 et les impacts des INP, avec plus de réalisme (figure 1). Ces éléments comprennent les compartiments pour les cas isolés et les contacts "exposés " mis en quarantaine à partir desquels la transmission aux personnes vulnérables est limitée ou absente, les compartiments pour les cas asymptomatiques qui peuvent être détectés par surveillance, ainsi que les flux vers les compartiments $d^{\prime}$ ' isolement » et de la " mise en quarantaine " qui permettent des variations selon les différents niveaux d'effort de santé publique. Les paramètres des modèles sont calibrés en fonction des valeurs obtenues par des recherches documentaires, qui sont effectuées chaque jour pour s'assurer que l'évolution des connaissances est saisie dans les modèles.

Figure 1 : Schéma du modèle de compartiments Analytica 5.3 montrant le flux de populations entre les compartiments

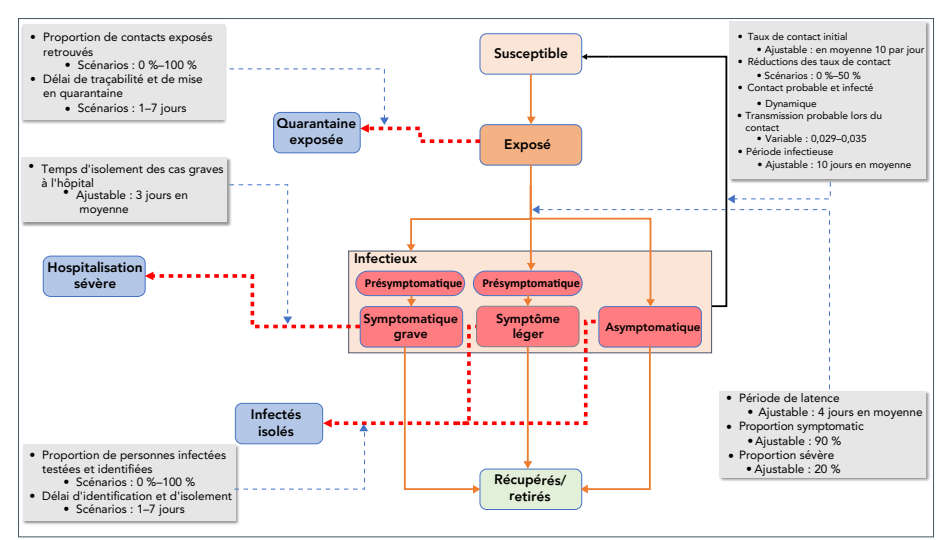

Abréviations : moy, moyenne; prob, probable

La flèche noire indique la transmission entre les populations infectées et vulnérables, les lignes rouges pointillées indiquent le retrait des personnes infectées ou exposées à la suite d'interventions non pharmaceutiques

Dans l'approche de modélisation déterministe, les effets de la distanciation physique sont modélisés de façon simple en réduisant les taux de contact quotidien par habitant fondés sur un certain nombre de paramètres, notamment les données sur les téléphones cellulaires (25). L'approche du modèle à base d'agents, qui contient des éléments stochastiques, utilise une représentation plus détaillée des communautés, notamment les maisons et les espaces de réunion communautaires qui peuvent représenter les lieux de travail, les écoles, les centres commerciaux et les restaurants, etc. Ce modèle est donc capable de modéliser les effets des fermetures de façon plus détaillée et de prédire différentes réalisations de la propagation de la maladie dans la communauté. L'approche à base d'agents comprend des taux de contact à l'intérieur et entre les groupes d'âge qui sont (20) fondés sur l'étude POLYMOD (26). Les données de contact propres au Royaume-Uni et aux pays européens ont été utilisées comme substitut des taux de contact au Canada, étant donné que des études semblables n'ont pas été réalisées au Canada. L'approche déterministe utilise un mélange homogène, mais elle est également modifiée pour inclure des taux de contact qui varient à l'intérieur des groupes d'âge et entre eux. À des fins d'illustration, un modèle de compartiment déterministe a été élaboré dans Analytica 5.3 (Lumina Inc.) en utilisant la connaissance de la transmission de la COVID-19 élucidée par les deux approches de modélisation. Le code de ce modèle est disponible sur demande avec des instructions sur la façon d'explorer le modèle et de générer des résultats.

Les principaux résultats des modèles sont les suivants : 1 ) dans quelles circonstances les INP provoquent l'extinction de l'épidémie en réduisant le taux de reproduction effectif $\left(R_{e}\right)$ en dessous de l'unité (c.-à-d. qu'une personne infectée infecte moins d'une autre personne, en moyenne), 2) le taux d'attaque final (c.-à-d. le pourcentage total de la population infectée) et 3) la durée approximative de l'épidémie.

\section{Résumé des résultats de la modélisation effectuée par l'Agence de la santé publique du Canada et d'autres groupes}

Le synopsis suivant des résultats des études de modélisation comprend celles réalisés par l'ASPC avec des référence aux études menées par des groupes de modélisation à l'extérieur du gouvernement du Canada. Les résultats sont illustrés ici à I'aide de graphiques générés par le modèle développé dans Analytica 5.3.

\section{Que se passe-t-il en l'absence d'INP?}

La base de référence pour comparer les effets des INP est un scénario dans lequel aucun effort n'est déployé pour contrôler la propagation de la maladie. Dans ce cas, le taux d'attaque devrait être supérieur à $70 \%$, et l'épidémie dure environ un an (figure 2). Ces résultats concordent avec ceux d'études estimant 
Figure 2 : Impacts des INP partiellement efficaces sur l'épidémie par rapport à la référence sans effort de contrôle

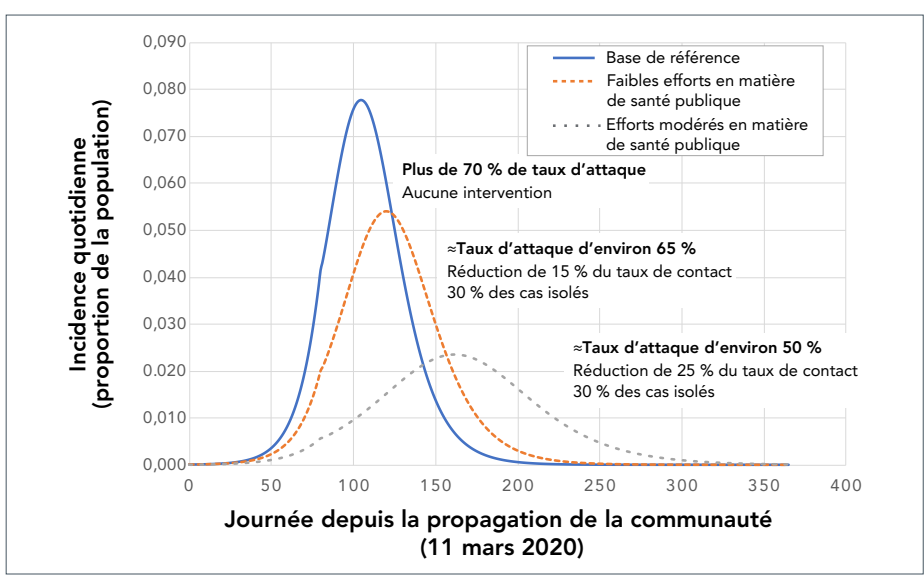

Les valeurs par courbe sont le taux d'attaque final estimé et les interventions non pharmaceutiques (INP) appliquées. On suppose que les INP sont en vigueur pendant toute la durée de l'épidémie

les répercussions sur les populations du Royaume-Uni et des États-Unis (11).

2. Que se passe-t-il lorsque les INP sont partiellement efficaces?

Si les INP, maintenues tout au long de l'épidémie, sont partiellement efficaces (c'est-à-dire qu'elles ont une incidence sur l'épidémie, sans l'éteindre), les principaux effets sont les suivants : l'épidémie est prolongée, le pic est réduit, la courbe épidémiologique est aplatie et le taux d'attaque est réduit à environ 50 \% (à $25 \%$ dans certains modèles) (20) (figure 2). Cette constatation concorde avec les études de modélisation équivalentes $(11,20)$. Ce scénario a été nommé « retard et réduction ".

Si les INP ne provoquent pas l'extinction de l'épidémie et sont retirées avant la fin de l'épidémie, il est prédit que la maladie reprendra en force et le taux d'attaque pourrait être aussi élevé que dans le scénario sans INP, parce que la majorité de la population reste naïve (figure 3 ) $(11,20)$.

\section{Que se passe-t-il lorsque les INP sont très efficaces?}

Lorsque les INP sont très efficaces, le taux $R_{e}$ tombe en dessous de l'unité, l'épidémie s'éteint et ne reprend pas de force si les INP sont retirées (aussi appelé lutte contre l'épidémie). La rapidité avec laquelle cela se produit, et donc le taux d'attaque final (qui peut se situer entre moins de $1 \%$ et $25 \%$ ), dépend de plusieurs facteurs, notamment le moment où les INP épidémiques sont mises en œuvre, l'intensité avec laquelle les INP sont mises en œuvre, la durée de la mise en œuvre et le niveau de conformité (figure 4) (12).
Figure 3 : Effet du lancement d'interventions non pharmaceutiques partiellement efficaces (dans ce cas, distanciation physique) et de l'élimination de ces interventions avant la fin de l'épidémie ${ }^{a}$

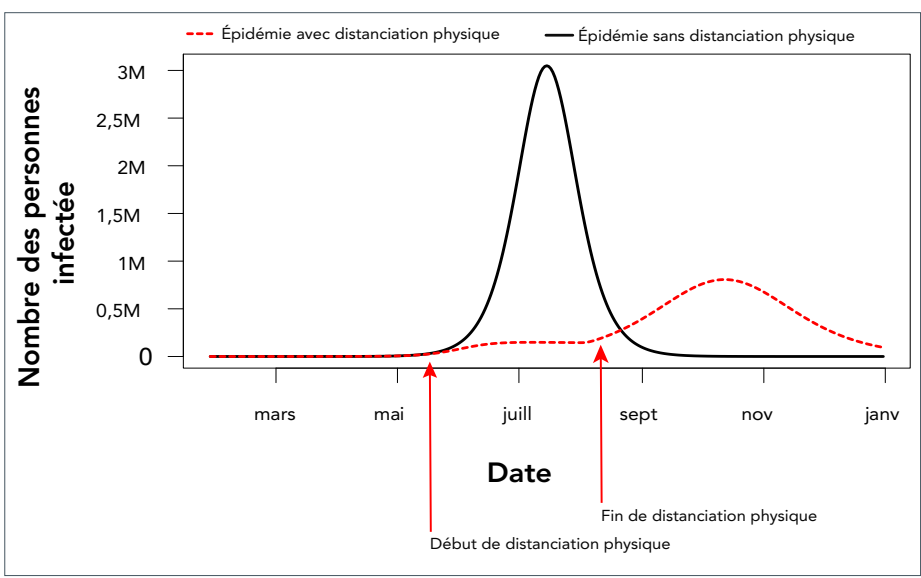

Estimations de la population canadienne d'Arino et de Portet (non publiées)

Figure 4 : Effets de différents niveaux et de différentes combinaisons d'interventions non pharmaceutiques sur le degré de maîtrise de l'épidémie et la rapidité avec laquelle elle est contrôlée

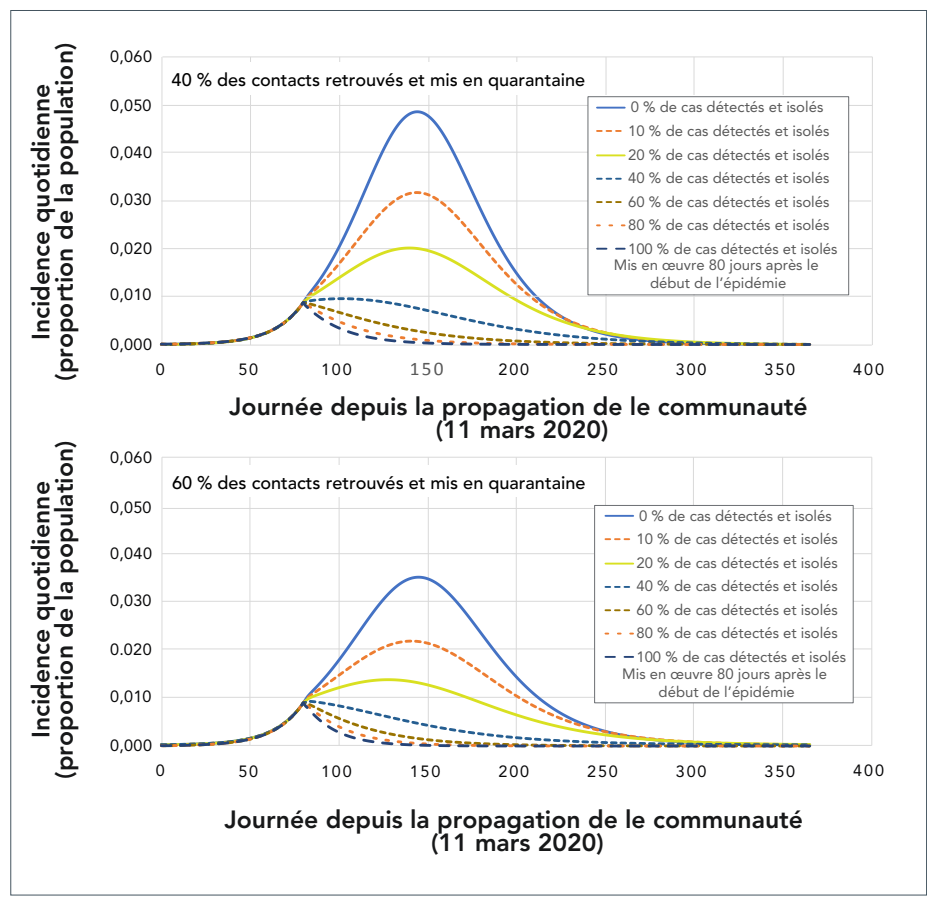

\section{Évaluation des taux d'hospitalisation et de mortalité à partir des taux d'attaque}

Le principal objectif des approches de modélisation était de comparer les impacts des différentes INP. II subsiste une grande incertitude quant à certains paramètres du modèle et à leur distribution, notamment la durée de la période de latence, la proportion de cas asymptomatiques et la durée de l'infectiosité. 
La force de ces modèles réside dans leur capacité à comparer entre différentes INP en utilisant les meilleures estimations actuelles des valeurs des paramètres. Toutefois, pour s'assurer que la capacité de soins de santé est suffisante pour répondre à la pandémie, les planificateurs doivent disposer d'une gamme d'estimations du nombre prévu de cas, d'hospitalisations, de cas nécessitant des soins dans les unités de soins intensifs (USI) et de décès. Les approches de modélisation initiales étaient axées sur l'estimation des taux d'attaque totaux et n'étaient pas conçues pour estimer les hospitalisations, les cas nécessitant des soins intensifs et les décès. Pour obtenir ces estimations à partir des taux d'attaque totaux, des estimations de gravité selon l'âge tirées de l'analyse des données de surveillance internationales (27) ont été utilisées pour évaluer la proportion de cas au Canada qui seraient bénins ou asymptomatiques, qui nécessiteraient une hospitalisation ou un traitement aux soins intensifs, et les cas pouvant entraîner un décès, selon la démographie de l'ensemble de la population canadienne (28). Les estimations par million d'habitants sont présentées au tableau 1.

Ces estimations sont brutes; des estimations plus précises des besoins en soins de santé devraient être calculées au niveau de la collectivité (e.g. la zone desservie par un hôpital ou un groupe d'hôpitaux) afin que la structure d'âge et les comorbidités particulières de la collectivité faisant l'objet de l'évaluation puissent être prises en compte dans le modèle (19).

\section{Observations au Canada et ailleurs dans le monde}

Les résultats des études de modélisation sont théoriques, mais leurs perspectives et leurs implications politiques ont été renforcées par des données concrètes. Le contrôle de l'épidémie a été réalisé à Singapour, en Chine et en Corée du Sud, avec un taux $R_{\mathrm{e}}$ en dessous de l'unité grâce à l'application d'un niveau rapide et intense d'INP $(5,21,29)$. En revanche, les INP en Europe ne semblent pas avoir ramené le taux $R_{e}$ en dessous de l'unité à ce jour (30). Au moment de la rédaction du présent rapport, l'épidémie est géographiquement hétérogène au Canada, mais des estimations non publiées donnent à penser que dans certains juridictions, le taux $R_{\mathrm{e}}$ peut être inférieur à
I'unité, tandis que cet état n'a pas été atteint dans d'autres régions (non publié; Ashleigh Tuite et David Champredon). Au moment de la rédaction du présent rapport, le taux de mortalité observé est plus élevé que celui prévu à l'aide des méthodes décrites ci-dessus en raison de la transmission étendue dans les établissements de soins de longue durée et les établissements pour personnes âgées. Dans ces établissements, les taux de contact sont probablement très élevés (31) et la population est très vulnérable à la COVID-19.

\section{Conclusion}

Les études de modélisation décrites ici fournissent des renseignements utiles à la planification des politiques de santé publique afin de lutter contre le risque sans précédent de la COVID-19 pour la santé et le bien-être de la population canadienne et mondiale. Ces études soulignent que sans les INP, la majorité de la population canadienne contracterait l'infection en relativement peu de temps et que le système de soins de santé serait fort probablement débordé, ce qui entraînerait un taux de mortalité plus élevé, particulièrement dans les groupes d'âge les plus vulnérables. L'intensité des INP et la conformité du public détermineront si l'épidémie est contrôlée, ou retardée et réduite. Le premier objectif semble être le meilleur, car le nombre de personnes touchées sera réduit au minimum. Cependant, ce scénario exigera un niveau très élevé d'efforts de santé publique et d'adhésion du public et, en cas de succès, il faudra un niveau élevé de vigilance pour identifier les cas importés et les chaînes de transmission qui pourraient en résulter, parce que la population canadienne demeurent en grande partie naïves relativement à l'infection. Si la transmission au Canada n'est pas complètement éteinte, il faudra maintenir de fortes INP, sinon l'épidémie reprendra. Toute levée de la distanciation physique, qui semble porter fruit à l'heure actuelle, devra être accompagnée d'efforts accrus pour détecter les cas par la surveillance et pour retracer les contacts afin de le mettre en quarantaine.

Les études de modélisation ne sont pas des prédictions; elles présentent des résultats plausibles avec différents niveaux d'INP, compte tenu de nos connaissances actuelles du virus et de sa transmission, et peuvent être utilisées pour appuyer la planification, en particulier dans des situations limitées par des données probantes, comme les épidémies émergentes de

Tableau 1 : Estimations par million d'habitants des hospitalisations, patients nécessitant des soins intensifs et décès selon différents scénarios de niveaux de contrôle ${ }^{a}$

\begin{tabular}{|c|c|c|c|c|c|c|}
\hline $\begin{array}{c}\text { Niveau de contrôle de } \\
\text { l'épidémie }\end{array}$ & \multicolumn{3}{|c|}{ « Retarder et réduire » } & \multicolumn{3}{|c|}{ Lutte contre l'épidémie } \\
\hline Taux d'attaque & $50 \%$ & $25 \%$ & $10 \%$ & $5 \%$ & $2,5 \%$ & $1 \%$ \\
\hline Tous les cas & 500000 & 250000 & 100000 & 50000 & 25000 & 10000 \\
\hline Léger (89,5 \%) & 450000 & 225000 & 90000 & 45000 & 22000 & 9000 \\
\hline Hospitalisés—pas de SI (8 \%) & 39000 & 19000 & 7800 & 3900 & 2000 & 800 \\
\hline Hospitalisés - SI (2,5 \%) & 12000 & 6000 & 2400 & 1200 & 600 & 200 \\
\hline Décès $(1,2 \%$ de tous les cas) & 6000 & 3000 & 1200 & 600 & 300 & 100 \\
\hline
\end{tabular}

Abréviation : $\mathrm{SI}$, soins intensifs

a Les chiffres supérieurs à 1000 ont été arrondis au millier le plus proche 
maladies infectieuses. Nos connaissances évoluent constamment, et les modèles et leurs résultats évoluent en conséquence. Les modèles fournissent des renseignements utiles pour la prise de décisions, mais ils ne dictent pas de décisions à prendre. Les décisions sur les programmes de santé publique visant à contrôler la COVID-19 au Canada tiendront compte d'un éventail de facteurs supplémentaires qui comprennent, sans s'y limiter, les répercussions économiques, les préoccupations éthiques et juridiques et les effets négatifs sur la santé de mesures agressives de distanciation physique.

\section{Financement}

Ce travail a été financé par l'Agence de la santé publique du Canada. Le travail de J. A. et de J. W. a également été financé par le programme de recherche rapide 2019 sur le nouveau coronavirus (COVID-19) des Instituts de recherche en santé du Canada (IRSC).

\section{Références}

1. Lau SK, Luk HK, Wong AC, Li KS, Zhu L, He Z, Fung J, Chan TT, Fung KS, Woo PC. Possible Bat Origin of Severe Acute Respiratory Syndrome Coronavirus 2. Emerg Infect Dis 2020;26(7). DOI [Publié en ligne avant impression] PubMed

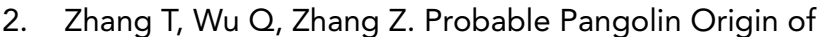
SARS-CoV-2 Associated with the COVID-19 Outbreak. Curr Biol 2020 Apr;30(7):1346-1351.e2. DOl PubMed

3. Andersen KG, Rambaut A, Lipkin WI, Holmes EC Garry RF. The proximal origin of SARS-CoV-2. Nat Med 2020;26(4):450-2. DOI PubMed

4. World Health Organization. WHO Timeline - COVID-19. Geneva (CH): WHO; April 27, 2020. https://www.who.int/ news-room/detail/08-04-2020-who-timeline---covid-19

5. Wang $C$, Liu L, Hao X, Guo H, Wang $Q$, Huang J, He N, Yu H, Lin X, Pan A, Wei S, Wu T. Association of Public Health Interventions With the Epidemiology of the COVID-19 Outbreak in Wuhan, China. JAMA 2020;323(19):1915-23. $\mathrm{DOI}$

6. Wu JT, Leung K, Leung GM. Nowcasting and forecasting the potential domestic and international spread of the 2019-nCoV outbreak originating in Wuhan, China: a modelling study. Lancet 2020;395(10225):689-97. DOI PubMed

7. Gouvernement du Canada. Maladie à coronavirus (COVID-19). Ottawa (ON); Gouvernement du Canada; mise à jour le 15 mai 2020. https://www.canada.ca/fr/ sante-publique/services/maladies/maladie-coronaviruscovid-19.html
8. Li J, Wang Y, Gilmour S, Wang M, Yoneoka D, Wang Y, You X, Gu J, Hao C, Peng L, Du Z, Xu DR, Hao Y. Estimation of the epidemic properties of the 2019 novel coronavirus: $A$ mathematical modeling study. MedRXiv: 2020a https://www. medrxiv.org/content/10.1101/2020.02.18.20024315v1.full. $\mathrm{pdf}$

9. Tang B, Wang X, Li Q, Bragazzi NL, Tang S, Xiao Y, Wu J. Estimation of the Transmission Risk of the 2019-nCoV and Its Implication for Public Health Interventions. J Clin Med 2020a;9(2):E462. DOI PubMed

10. Kucharski AJ, Russell TW, Diamond C, Liu Y, Edmunds J, Funk S, Eggo RM; Centre for Mathematical Modelling of Infectious Diseases COVID-19 Working Group. Early dynamics of transmission and control of COVID-19: a mathematical modelling study. Lancet Infect Dis. 2020;20(5)552-8. DOI [Publié en ligne avant impression]

11. Ferguson NM, Laydon D, Nedjati-Gilani G, Imai N, Ainslie K, Baguelin M, Bhatia S, Boonyasiri A, Cucunubá Z, Cuomo-Dannenburg G, Dighe A, Dorigatti I, Fu H, Gaythorpe K, Green W, Hamlet A, Hinsley W, Okell LC, van Elsland S, Thompson H, Verity R, Volz E, Wang H, Wang Y, Walker PG, Walters C, Winskill P, Whittaker C, Donnelly CA, Riley S, Ghani AC. Impact of non-pharmaceutical interventions (NPIs) to reduce COVID-19 mortality and healthcare demand. London (UK): Imperial College; 2020. https://www.imperial.ac.uk/media/imperial-college/ medicine/sph/ide/gida-fellowships/Imperial-CollegeCOVID19-NPI-modelling-16-03-2020.pdf

12. Hellewell J, Abbott $\mathrm{S}$, Gimma A, Bosse $\mathrm{NI}$, Jarvis $\mathrm{Cl}$, Russell TW, Munday JD, Kucharski AJ, Edmunds WJ, Funk S, Eggo RM; Centre for the Mathematical Modelling of Infectious Diseases COVID-19 Working Group. Feasibility of controlling COVID-19 outbreaks by isolation of cases and contacts. Lancet Glob Health 2020;8(4):e488-96. DOl PubMed

13. Wei WE, Li Z, Chiew CJ, Yong SE, Toh MP, Lee VJ. Presymptomatic Transmission of SARS-CoV-2 - Singapore, January 23-March 16, 2020. MMWR Morb Mortal Wkly Rep 2020;69(14):411-5. DOl PubMed

14. Vetter P, Vu DL, L'Huillier AG, Schibler M, Kaiser L, Jacquerioz F. Clinical features of covid-19. BMJ 2020;369:m1470. DOI PubMed

15. Wilder-Smith A, Chiew CJ, Lee VJ. Can we contain the COVID-19 outbreak with the same measures as for SARS? Lancet Infect Dis 2020 May;20(5):e102-7. DOI PubMed

16. Sanche S, Lin YT, Xu C, Romero-Severson E, Hengartner N, Ke R. High Contagiousness and Rapid Spread of Severe Acute Respiratory Syndrome Coronavirus 2. Emerg Infect Dis 2020;26(7). DOI [Publié en ligne avant impression] PubMed

17. Arino J, Brauer $F$, van den Driessche $P$, Watmough J, Wu J. A model for influenza with vaccination and antiviral treatment. J Theor Biol 2008;253(1):118-30. DOl PubMed

18. Tuite A, Fisman DN, Kwong JC, Greer A. Optimal pandemic influenza vaccine allocation strategies for the canadian population. PLoS Curr 2010;2:RRN1144. DOI PubMed 
19. Arino J, Portet S. A simple model for COVID-19. Inf Dis Modelling. 2020:5: 309-15. PubMed

20. Tuite AR, Fisman DN, Greer AL. Mathematical modelling of COVID-19 transmission and mitigation strategies in the population of Ontario, Canada. CMAJ 2020;192(19): E497-505. DOl PubMed

21. Li J, Yuan P, Heffernan J, Zheng T, Ogden N, Sander B, Li J, Li Q, Bélair J, Dzevela Kong J, Aruffo E, Tan Y, Jin Z, Yu Y, Fan M, Cui J, Teng Z, Zhu H. Observation wards and control of the transmission of COVID-19 in Wuhan. Bull World Health Organ. DOI

22. Ng V, Fazil A, Waddell S, Bancej C, Turgeon P, Ogden NH. An agent-based model of COVID-19 transmission in Canada: forecasting impacts and de-escalation of non-pharmaceutical public health interventions [soumis pour publication].

23. Ludwig A, Berthiaume $P$, Orpana $H$, Swerdfeger $H$, Otten A, Statistics Canada team, Ogden NH. A dynamic compartmental model of national COVID-19 transmission and the impact of varying levels of case identification and contact tracing in Canada [soumis pour publication].

24. R Core Team. R: A language and environment for statistical computing. Vienna (Austria): R Foundation for Statistical Computing; 2020. https://www.r-project.org/

25. Google. https://www.google.com/covid19/mobility/

26. Mossong J, Hens $\mathrm{N}$, Jit M, Beutels $P$, Auranen $\mathrm{K}$, Mikolajczyk R, Massari M, Salmaso S, Tomba GS, Wallinga J, Heijne J, Sadkowska-Todys M, Rosinska M, Edmunds WJ. Social contacts and mixing patterns relevant to the spread of infectious diseases. PLoS Med 2008;5(3):e74. DOI PubMed

27. Verity R, Okell LC, Dorigatti I, Winskill P, Whittaker C, Imai N, Cuomo-Dannenburg G, Thompson H, Walker PGT, Fu H, Dighe A, Griffin JT, Baguelin M, Bhatia S, Boonyasiri A, Cori A, Cucunubá Z, FitzJohn R, Gaythorpe K, Green W, Hamlet A, Hinsley W, Laydon D, Nedjati-Gilani G, Riley S, van Elsland S, Volz E, Wang H, Wang Y, Xi X, Donnelly CA, Ghani AC, Ferguson NM. Estimates of the severity of coronavirus disease 2019: a model-based analysis. Lancet Infect Dis 2020:pii:S1473-3099(20)30243-7. DOl [Publié en ligne avant impression]
28. Statistique Canada. Estimations de la population au $1^{\mathrm{er}}$ juillet, par âge et sexe. Tableau 17-10-0005-01. Ottawa (ON) : StatsCan; mise à jour le 18 mai 2020. https://www150. statcan.gc.ca/t1/tb/1/fr/tv.action?pid=1710000501\&reque st_locale $=\mathrm{fr}$

29. Tang B, Xia F, Bragazzi NL, McCarthy Z, Wang X, He S, Sun X, Tang S, Xiao Y, Wu J. Lessons drawn from China and South Korea for managing COVID-19 epidemic: insights from a comparative modeling study. Bull World Health Organ 2020. DOI [Publié en ligne avant impression]

30. Flaxman S, Mishra S, Gandy A, Unwin HJ, Coupland $H$, Mellan TA, Zhu H, Berah T, Eaton JW, Guzman PN, Schmit N, Callizo L, Ainslie KE, Baguelin M, Blake I, Boonyasiri A, Boyd O, Cattarino L, Ciavarella C, Cooper L, Cucunubá Z, Cuomo-Dannenburg G, Dighe A, Djaafara B, Dorigatti I, van Elsland S, FitzJohn R, Fu H, Gaythorpe K, Geidelberg L, Grassly N, Green W, Hallett T, Hamlet A, Hinsley W, Jeffrey B, Jorgensen D, Knock E, Laydon D, Nedjati-Gilani G, Nouvellet $P$, Parag K, Siveroni I, Thompson H, Verity R, Volz E, Walters C, Wang H, Wang Y, Watson O, Winskill P, Xi X, Whittaker C, Walker PG, Ghani A, Donnelly CA, Riley S, Okell LC, Vollmer MA, Ferguson NM, Bhatt S. Report 13: Estimating the number of infections and the impact of non-pharmaceutical interventions on COVID-19 in 11 European countries. London (UK): Imperial College; 2020. https://www.imperial.ac.uk/mrc-global-infectious-diseaseanalysis/covid-19/report-13-europe-npi-impact/

31. Najafi M, Laskowski M, de Boer PT, Williams E, Chit A, Moghadas SM. The effect of individual movements and interventions on the spread of influenza in long-term care facilities. Med Decis Making 2017;37(8):871-81. DOI PubMed 